discontinuation. These data suggest that the development of ADA is a frequent cause of secondary inefficacy in our RA pts

Disclosure of Interest: None declared

DOI: 10.1136/annrheumdis-2017-eular.6371

\section{SAT0169 MAINTENANCE AND IMPROVEMENT IN CLINICAL EFFICACY BETWEEN WEEK 12 AND 24 IN PATIENTS WITH RHEUMATOID ARTHRITIS TREATED WITH SB4 OR REFERENCE ETANERCEPT}

$\underline{\text { P. Emery }}^{1}$, J. Vencovsky ${ }^{2}$, E. Keystone ${ }^{3}$, J. Ghil ${ }^{4}$, S.Y. Cheong ${ }^{4}$, E.E. Hong ${ }^{4}$. ${ }^{1}$ Leeds Institute of Rheumatic and Musculoskeletal Medicine, Leeds, United Kingdom; ${ }^{2}$ Institute of Rheumatology, Prague, Czech Republic; ${ }^{3}$ Mount Sinai Hospital, Toronto, Canada; ${ }^{4}$ Samsung Bioepis Co., Ltd., Incheon, Korea, Republic Of

Background: SB4 is approved by the European Commission as a biosimilar of the reference etanercept (ETN).

Objectives: To evaluate the maintenance and improvement in clinical efficacy between week 12 and 24 in patients with rheumatoid arthritis (RA) treated with SB4 or ETN from a post-hoc analysis of phase III results.

Methods: Patients with RA were randomised to receive $50 \mathrm{mg} /$ week of either SB4 or ETN with background methotrexate. American College of Rheumatology (ACR) and European League Against Rheumatism (EULAR) responses were compared at week 12 and week 24. At week 12, patients with available assessment results were categorised as ACR responders or ACR non-responders and EULAR responders (patients with moderate or good EULAR response) or EULAR nonresponders. The same was assessed for week 24 and patients with missing data at week 24 were regarded as non-responders.

Results: A total of 551 patients (283 patients from SB4 and 268 patients from ETN) completed 24 weeks of the study. In both treatment groups, efficacy was well maintained between week 12 and week 24. Among patients who were ACR20, 50, or 70 responders at week $12,90.8 \%$ vs. $91.4 \%, 80.5 \%$ vs. $80.6 \%$, and 74.5 vs. $77.5 \%$ of patients from SB4 and ETN, respectively, maintained their responses at week 24. Likewise, EULAR response was maintained by $93.1 \%$ vs. $92.6 \%$ of patients who had a good or moderate response at week 12. (Table). The improvement in ACR responses between week 12 and 24 was comparable between SB4 and ETN group (Table). In SB4 and ETN, respectively, 42.1\% vs. $50.5 \%$ of 12 -week ACR20 non-responders became ACR20 responders at week 24 . Similarly, $20.9 \%$ vs. $21.9 \%$ of 12 -week ACR50 non-responders became ACR50 responders and $13.0 \%$ vs. $11.4 \%$ of 12 -week ACR70 non-responders became ACR70 responders. The improvement in EULAR responses was also comparable between SB4 and ETN. $43.2 \%$ vs. $52.2 \%$ of 12 -week EULAR nonresponders in SB4 and ETN, respectively, became EULAR responders at week 24.

Table. Maintenance and improvement of efficacy between week 12 and week 24

\begin{tabular}{|l|l|c|c|}
\hline \multirow{2}{*}{} & & \multicolumn{2}{|c|}{ Week 24 responders } \\
\cline { 3 - 4 } & & $\begin{array}{c}\text { SB4 50 mg } \\
\mathbf{n} / \mathbf{n} \text { ' (\%) }\end{array}$ & $\begin{array}{c}\text { ETN 50 mg } \\
\mathbf{n} / \mathbf{n} \text { ' (\%) }\end{array}$ \\
\hline \multirow{2}{*}{ ACR20 } & Responder at week 12 & $179 / 196(91.3 \%)$ & $162 / 175(92.6 \%)$ \\
& Non-responder at week 12 & $41 / 97(42.3 \%)$ & $51 / 105(48.6 \%)$ \\
\hline \multirow{2}{*}{ ACR50 } & Responder at week 12 & $91 / 114(79.8 \%)$ & $75 / 93(80.6 \%)$ \\
& Non-responder at week 12 & $37 / 179(20.7 \%)$ & $41 / 187(21.9 \%)$ \\
\hline \multirow{2}{*}{ ACR70 } & Responder at week 12 & $38 / 51(74.5 \%)$ & $31 / 40(77.5 \%)$ \\
& Non-responder at week 12 & $31 / 242(12.8 \%)$ & $28 / 240(11.7 \%)$ \\
\hline \multirow{2}{*}{ EULAR response* } & Responder at week 12 & $231 / 246(93.9 \%)$ & $215 / 231(93.1 \%)$ \\
& Non-responder at week 12 & $19 / 46(41.3 \%)$ & $24 / 49(49.0 \%)$ \\
\hline \multirow{2}{*}{ n, number of responders; n', number of responders or non-responders at week 12 } \\
"Patients with moderate or good EULAR response were regarded as responders.
\end{tabular}

Conclusions: Efficacy of SB4 and ETN was well maintained and the maintenance rate was comparable between week 12 and week 24 . In addition, a similar and considerable proportion of patients in SB4 and ETN who did not achieve a clinical response at week 12 reached clinical response at week 24 . These results suggest that etanercept non-responders at week 12 may benefit from continuing treatment up to 24 weeks.

Disclosure of Interest: P. Emery Grant/research support from: Abbvie, BMS, Pifzer, USB, MSD, Novartis, Lilly, Consultant for: Samsung Bioepis Co., Ltd., J. Vencovsky Consultant for: Samsung Bioepis Co., Ltd., Biogen, E. Keystone Grant/research support from: Abbott, Amgen, AstraZeneca, BMS, Janssen, Lilly, Novartis, Pfizer, Consultant for: Abbott, AstraZeneca, Biotest, BMS, Genentech, Janssen, Lilly, Merck, Pfizer, Samsung Bioepis, Speakers bureau: Abbott, AstraZeneca, BMS Canada, Janssen, J. Ghil Employee of: Samsung Bioepis Co., Ltd., S. Y. Cheong Employee of: Samsung Bioepis Co., Ltd., E. Hong Employee of: Samsung Bioepis Co., Ltd. DOI: 10.1136/annrheumdis-2017-eular.3344

\section{SAT0170 B-CELL PHENOTYPE AND IGD-CD27- MEMORY B CELLS ARE AFFECTED BY TNF-INHIBITORS AND TOCILIZUMAB TREATMENT IN RHEUMATOID ARTHRITIS}

R.A. Moura ${ }^{1}$, C. Quaresma ${ }^{1}$, A.R. Vieira ${ }^{1}$, M.J. Gonçalves ${ }^{1,2}$, J. Polido-Pereira ${ }^{1,2}$, V.C. Romão ${ }^{1,2}$, N. Martins ${ }^{2}$, H. Canhão ${ }^{1,2}$, J.E. Fonseca ${ }^{1,2}$ ${ }^{1}$ Rheumatology Research Unit, Instituto de Medicina Molecular; ${ }^{2}$ Rheumatology Department, Centro Hospitalar de Lisboa Norte, EPE, Hospital de Santa Maria, Lisbon, Portugal

Background: The use of TNF-inhibitors and/or the IL-6 receptor antagonist, tocilizumab, in rheumatoid arthritis (RA) have pleiotropic effects that also involve circulating B-cells.

Objectives: The main goal of this study was to assess the effect of TNF-inhibitors and tocilizumab on B-cell phenotype and gene expression in RA.

Methods: Blood samples were collected from untreated early RA (ERA) patients $(<1$ year of disease duration), established RA patients under methotrexate treatment, established RA patients before and after treatment with TNF-inhibitors and tocilizumab, and healthy donors. B-cell subpopulations were characterized by flow cytometry and B-cell gene expression was analyzed by real-time PCR on isolated B-cells. Serum levels of BAFF, CXCL13 and SCD23 were determined by ELISA.

Results: The frequency of total CD19+ B-cells in circulation was similar between controls and all RA groups, irrespective of treatment, but double negative (DN) IgD-CD27- memory B-cells were significantly increased in ERA and established RA when compared to controls. Treatment with TNF-inhibitors and tocilizumab restored the frequency of IgD-CD27- B-cells to normal levels, but did not affect other B-cell subpopulations. TACI, CD95, CD5, HLA-DR and TLR9 expression on B-cells significantly increased after treatment with either TNF-inhibitors and/ or tocilizumab, but no significant changes were observed in BAFF-R, BCMA, CD69, CD86, CXCR5, CD23, CD38 and IgM expression on B-cells when comparing baseline with post-treatment follow-ups. Alterations in B-cell gene expression of BAFF-R, TACI, TLR9, Fc $\gamma R$ RIIB, BCL-2, BLIMP-1 and $\beta 2 \mathrm{M}$ were found in ERA and established RA patients, but no significant differences were observed after TNF-inhibitors and tocilizumab treatment when comparing baseline and follow-ups. Serum levels of CXCL13, sCD23 and BAFF were not significantly affected by treatment with TNF-inhibitors and tocilizumab.

Conclusions: In RA, treatment with either TNF-inhibitors or tocilizumab affects B-cell phenotype and the frequency of memory B-cell subpopulations in peripheral blood, particularly DN (IgD-CD27-) B-cells, but not B-cell gene expression or serum levels of CXCL13, SCD23 and BAFF, when comparing baseline with post-treatment follow up. Overall, our results may suggest that TNF-inhibitors and tocilizumab inhibit B-cell trafficking towards inflammatory sites, thus supporting activated B-cell recirculation from tissues through blood and lymphatic systems.

Disclosure of Interest: None declared

DOI: 10.1136/annrheumdis-2017-eular.3011

\section{SAT0171 ABP 501 BIOSMILAR TO ADALIMUMAB: FINAL SAFETY, IMMUNOGENICITY, AND EFFICACY RESULTS FROM AN OPEN-LABEL EXTENSION STUDY}

S. Cohen ${ }^{1}$, J.L. Pablos ${ }^{2}$, H. Wang ${ }^{3}$, G.A. Müller ${ }^{4}$, A. Kivitz ${ }^{5}$, A. Matsumoto ${ }^{6}$ E. Krishnan ${ }^{3}$. ${ }^{1}$ Metroplex Clinical Research Center, Dallas, United States; ${ }^{2}$ Instituto de Investigación Hospital, Madrid, Spain; ${ }^{3}$ Amgen, Thousand Oaks, United States; ${ }^{4}$ Abteilung für Nephrologie und Rheumatologie, Göttingen, Germany; ${ }^{5}$ Altoona Center for Clinical Research, Duncansville; ${ }^{6}$ Arthritis and Rheumatism Associates, Wheaton, United States

Background: ABP 501 has been approved by the US FDA as the first biosimilar to the fully human recombinant monoclonal antibody, adalimumab. Totality of evidence to date suggests that ABP 501 is highly similar to adalimumab. Subjects receiving either ABP 501 or adalimumab in the active-controlled, comparative, pivotal phase 3 study in rheumatoid arthritis (parent study) continued on to this open-label extension (OLE) study if they had completed the final week 26 visit of that study.

Objectives: To describe the safety, immunogenicity, and efficacy outcomes of ABP 501 in the OLE study.

Methods: Subjects who completed the parent study were screened and were included if they met the eligibility requirements. All subjects included in the OLE were treated with ABP $50140 \mathrm{mg}$ subcutaneously every other week for 68 weeks followed by disease assessments at week 70 and the follow-up safety assessment at week 72 (or early termination). Data were summarized descriptively and no inferential analyses were performed.

Results: Of the 467 subjects enrolled in the OLE study, 466 were treated with ABP 501. Of these, 237 transitioned from the adalimumab arm of the parent study; 412/467 completed the study. Demographics and disease characteristics were balanced between subjects who transitioned from adalimumab and those who continued on ABP 501 from the parent study. Overall, the incidence of treatmentemergent adverse events (TEAEs) was $63.7 \%$ (297/466) and that of grade $\geq 3$ TEAEs was $9.0 \%$ (42/466); incidence of TEAEs leading to discontinuation of investigational product was $3.6 \%(17 / 466)$. TEAEs with incidence $\geq 5 \%$ were nasopharyngitis $(9.2 \%)$, upper respiratory tract infection $(8.6 \%)$, bronchitis $(6.4 \%)$, rheumatoid arthritis $(6.2 \%)$, hypertension $(4.7 \%)$, and pharyngitis $(4.1 \%)$. The incidence of serious adverse events was $9.9 \%$ (46/466). Most common 
TEAEs of interest were infections (40.8\%), liver enzyme elevations $(5.4 \%)$, and hypersensitivity $(4.3 \%)$.

Overall, $18.2 \%$ (85/466) of subjects developed binding antidrug antibodies (ADAs) and $6.9 \%(32 / 466)$ developed neutralizing ADAs in the OLE study. The rates of ADA formation were similar between subjects who transitioned from adalimumab and those who continued on ABP 501.

The ACR20 response rate (using the parent study baseline) was 73.3\% (340/464) at the OLE study baseline, $77.6 \%(361 / 465)$ at week $4,74.2 \%(336 / 453)$ at week $24,77.6 \%(337 / 434)$ at week 48 , and $78.8 \%(327 / 415)$ at week 70 . The overall mean DAS28-CRP change from parent study baseline was $-2.25(n=440)$ at the OLE study baseline, $-2.36(n=463)$ at week $4,-2.41(n=450)$ at week $24,-2.55$ at week $48(n=433)$, and -2.60 at week $70(n=412)$.

Conclusions: In this OLE study of ABP 501, efficacy documented in the parent study was maintained with no new safety findings. Long-term safety, immunogenicity, and efficacy results were similar between subjects who transitioned from adalimumab and those who continued on ABP 501 from the parent study.

Disclosure of Interest: S. Cohen Consultant for: Amgen Inc, J. L. Pablos Consultant for: Amgen Inc, H. Wang Shareholder of: Amgen Inc, Employee of: Amgen Inc, G. Müller Consultant for: Amgen Inc, A. Kivitz Consultant for: Amgen Inc, A. Matsumoto Consultant for: Amgen Inc, E. Krishnan Shareholder of: Amgen Inc, Employee of: Amgen Inc

DOI: 10.1136/annrheumdis-2017-eular.3288

\section{SAT0172 ECONOMIC OUTCOMES, TREATMENT PATTERNS, AND ADVERSE EVENTS AND REACTIONS FOR PATIENTS PRESCRIBED INFLIXIMAB OR CT-P13 IN THE TURKISH POPULATION}

K. Phillips ${ }^{1}$, T. Juday ${ }^{2}$, Q. Zhang ${ }^{3}$, A. Keshishian ${ }^{3} \cdot{ }^{1}$ Vanderbilt University Medical Center, Nashville; ${ }^{2}$ AbbVie Inc., North Chicago; ${ }^{3}$ STATinMED Research, Ann Arbor, United States

Background: CT-P13, a biosimilar drug product to infliximab, was approved and marketed in July 2014 in Turkey. There is little information on the costs, treatment discontinuation and adverse events and reactions between patients who switched from infliximab to CT-P13 and patients who continued infliximab.

Objectives: The study objective was to evaluate health care costs, treatment discontinuation, and adverse events and reactions between patients who switched from infliximab to CT-P13 and patients who continued infliximab in the Turkish population.

Methods: Adult patients with $>1$ claim for infliximab or CT-P13 were identified in a Turkish healthcare administrative database representing $80 \%$ of the Turkish population during the identification period (16 July 2014-31 Aug 2015). Patients were required to continuously use infliximab for $>6$ months with no hospitalizations. Eligible patients either continued on infliximab (index date: date of first infliximab prescription), or switched from infliximab to CT-P13 (index date: switch date). Patients were excluded if they had $>1$ condition with an indication for infliximab during the baseline period. Patients who switched to CT-P13 were 1:10 matched to patients who continued infliximab based on the length of infliximab use prior to the index date. Demographics and clinical characteristics were measured 12 months pre-index date. Generalized linear models were used to compare adjusted health care costs, Cox regression was used to evaluate the adjusted risk of discontinuation, and Poisson regression was used to evaluate the adjusted risk of adverse events and reactions.

Results: The study included 1,524 patients, of whom 1,388 were continuous infliximab users and 136 switched to CT-P13. Ankylosing spondylitis and rheumatoid arthritis were the most common conditions indicated for infliximab and CT-P13; however, patients were much less likely to be switched to CT-P13 for other conditions. After adjusting for demographics and clinical characteristics, patients who switched to CT-P13 had higher outpatient ([Turkish lira] TL 269 vs TL 181; $p=0.005$ ), inpatient (TL 64 vs TL 29; $p=0.313$ ), and pharmacy costs (TL 1,473 vs TL 1,$329 ; p=0.371$, which resulted in significantly higher total health care costs ( $T L 2,009$ vs $T L 1,640 ; p=0.046$ ) compared to patients who continued infliximab. Additionally, patients who switched to CT-P13 were more likely to discontinue treatment (13.2 vs 1.52 per 1000 person-years) compared to those who continued infliximab. Of patients who discontinued CT-P13, 79\% switched back to infliximab. After adjusting for baseline characteristics, patients who switched to CT-P13 were significantly more likely to discontinue treatment compared to those who continued infliximab ( $\mathrm{HR}=5.53 ; 95 \% \mathrm{Cl}: 4.01-7.63)$. There was no difference in the adjusted incidence rate ratio (IRR) between the cohorts for adverse events (IRR=0.67; 95\% Cl: $0.19-2.30$ ) and reactions (IRR $=0.84 ; 95 \% \mathrm{Cl}: 0.55-1.27$ ).

Conclusions: Patients who switched to CT-P13 had significantly higher health care costs and were also more likely to discontinue treatment compared to those who continued infliximab. However, there was no difference in the rate of adverse events and reactions.

Disclosure of Interest: K. Phillips Consultant for: AbbVie Inc., T. Juday Shareholder of: AbbVie Inc., Employee of: AbbVie Inc., Q. Zhang Employee of: STATinMED Research, A. Keshishian Employee of: STATinMED Research

DOI: 10.1136/annrheumdis-2017-eular.2933

\section{SAT0173 SWITCHING FROM REFERENCE PRODUCT ETANERCEPT TO THE BIOSIMILAR SB4 IN A REAL-LIFE SETTING: FOLLOW-UP OF 147 PATIENTS}

V. Sigurdardottir ${ }^{1,2}$, T. Husmark ${ }^{1}$, A. Svärd ${ }^{1,2} \cdot{ }^{1}$ Reumatologkliniken, Falu lasarett; ${ }^{2}$ Centre of Clinical Research Dalarna, Falun, Sweden

Background: The etanercept biosimilar SB4 was introduced in Sweden in early 2016. SB4 has been shown in a randomized controlled trial to be equivalent to its etanercept reference product (ERP) in terms of efficacy and safety in subjects with active rheumatoid arthritis (RA) (1). In light of this, and the fact that biosimilars offer considerable cost savings, all patients being treated with ERP at our clinic were switched to treatment with SB4 in april 2016.

Objectives: To describe the clinical experiences of switching patients on treatment with ERP to SB4 at our clinic.

Methods: All patients using ERP $50 \mathrm{mg}$ at our clinic were identified using the Swedish Rheumatology Quality Register (SRQ). The patients were issued prescriptions for SB4 $50 \mathrm{mg}$ and were sent a letter encouraging them to switch to SB4 when they ran out of ERP. The process of switching was started 21st. However, the actual date of starting treatment with SB4 might have been 0-90 days from April 20th for individual patients, as prescriptions are written in 3 month increments.

Patients were followed up clinically and in the SRQ as planned at the last visit before switching.

For RA and psoriatic arthritis (PsA) patients DAS28 values from the last visit preceding the switch and the last visit after the switch registered up to January 2017 were collected from the SRQ. The paired T-test was used to compare mean DAS28 before and after switching.

Results: Before the switch, 147 patients were on treatment with ERP. Indications for treatment were RA $(\mathrm{N}=76)$, PsA $(\mathrm{N}=28)$, other spondyloarthritis $(\mathrm{N}=13)$, ankylosing spondylitis $(\mathrm{N}=12)$, unspecified arthritis $(\mathrm{N}=10)$ and juvenile arthritis $(\mathrm{N}=8)$.

At the end of January 2017, 126 patients (86\%) were still on SB4.

Since the switch, 9 patients have requested to be switched back to ERP, 2 made the request before initiating therapy with SB4. No objective evidence for lack of efficacy was seen in these 9 patients. Seven patients have stopped treatment with SB4 because of inactive disease. Five patients have been switched to a non-etanercept biologic because of lack of efficacy, these patients also had lack of efficacy when on ERP.

The 76 RA patients had a mean disease duration of 17 years and had been on ERP for a mean duration of 4.7 years. As of January 2017, 60 of the RA patients have been on a follow-up visit and 54 of these had available DAS28 data from both the last visit before and after switching. For the RA patients DAS28 was 2.80 before and 2.79 after switching, $p=0.960$. Complete DAS28 data was available for 23 of the 28 PsA patients, mean DAS28 was 2.54 before switching and 2.06 after, $p=0.161$. The mean duration since switching at follow-up was 22 weeks for the RA and PsA patients.

Conclusions: Switching from the etanercept reference product to the biosimilar SB4 was acceptable to most of our patients. Low mean disease activity has been maintained in the RA and PSA group after the switch.

\section{References:}

[1] Emery P, Vencovsky J, Sylwestrzak A, Leszczynski P, Porawska W, Baranauskaite A, et al. A phase III randomised, double-blind, parallelgroup study comparing SB4 with etanercept reference product in patients with active rheumatoid arthritis despite methotrexate therapy. Annals of the Rheumatic Diseases. 2017;76(1):51-7.

Disclosure of Interest: None declared

DOI: 10.1136/annrheumdis-2017-eular.4391

\section{SAT0174 USE OF A 8-WEEK OBSERVATIONAL PERIOD FOR PREDICTING REMISSION AND LOW DISEASE ACTIVITY AT 52 WEEKS IN RA PATIENTS TREATED WITH CERTOLIZUMAB PEGOL - A MULTICENTER STUDY}

Y. Kanayama ${ }^{1}$, A. Kaneko ${ }^{2}$, N. Takahashi ${ }^{3}$, T. Kato ${ }^{4}$, M. Hayashi ${ }^{5}$, Y. Hattori ${ }^{2}$, N. Asai ${ }^{3}$, N. Ishiguro ${ }^{3}$, T. Kojima ${ }^{3}$ on behalf of TBCR study group. ${ }^{1}$ Orthopedic Surgery and Rheumatology, Toyota Kosei Hospital, Toyota; ${ }^{2}$ Orthopedic Surgery and Rheumatology, Nagoya Medial Center; ${ }^{3}$ Orthopedic Surgery, Nagoya University Graduate school of Medicine, Nagoya; ${ }^{4}$ Orthopedic Surgery, Kato clinic, Okazaki; ${ }^{5}$ Orthopedic Surgery and Rheumatology, Nagano Red Cross Hospital, Nagano, Japan

Background: Certolizumab pegol (CZP) is a polyethylene glycol (PEG)ylated Fc-free new anti-TNF $\alpha$ agent. However few data still reported clinical efficacy of CZP treatment in the routine practice.

Objectives: This study aimed to provide clinical evidence of an adequate observational period for predicting remission and low disease activity (LDA) achievement at 52 weeks in RA patients treated with Certolizumab pegol (CZP). Methods: Patients with a diagnosis of RA according to the 2010 ACR/EULAR criteria who had been prescribed CZP from Tsurumai Biologics Communication Registry (TBCR) between May 2013 and October 2015 were enrolled.The final study cohort of 98 Japanese RA patients. We reviewed the methods about the improvement of DAS28-ESR and SDAl which was an index of disease activity of RA using Wilcoxon signed-rank test and the rate of remission and LDA patients at 